\title{
Functional Task Limitations in Obese Adults
}

\author{
Wendy Gilleard
}

Published online: 21 June 2012

(C) Springer Science+Business Media, LLC 2012

\begin{abstract}
Obesity is characterized by increased dimensions and mass. Functional task limitations and changes in the mechanical strategies used to complete functional tasks may be related independently to increased mass or increased dimensions. Increased mass may not always be detrimental. The mechanical strategies may vary with the class of obesity or mass distribution to the trunk or lower limbs. Much of the literature is equivocal and it is possible that methodological approaches and class of obesity of participants result in different or conflicting outcomes.
\end{abstract}

Keywords Obesity $\cdot$ Standing $\cdot$ Lateral bending $\cdot$ Forward flexion · Sit to stand · Posture · Walking · Lifting · Functional task $\cdot$ Pain $\cdot$ Kinematics $\cdot$ Kinetics

\section{Introduction}

Obesity is often identified as a factor associated with functional task limitations [1-4] and is viewed as detrimental; however, the etiology of the mechanical effects of obesity is not well understood. Obesity is characterized by increased dimensions and mass, both of which may alter the ability to undertake daily activities $[1,2,5,6]$ and modify the movement strategies used for successful completion. Daily activities consist of those we would like to do and those we do undertake in our lives at home, during recreation, and in employment and therefore the impact of obesity is important to consider. Functional tasks, as the building blocks of daily

\section{W. Gilleard $(\bowtie)$}

Southern Cross University,

Military Rd,

Lismore, NSW 2480, Australia

e-mail: Wendy.Gilleard@scu.edu.au activities, can be utilized to consider the mechanical effects of obesity on functional task limitations and the strategies utilized in the daily lives of adults who are obese.

\section{Spinal Curvatures During Quiet Standing Posture}

Obesity is often thought to be associated with poor standing spinal morphological posture; however, the literature is equivocal. Using surface measurement techniques such as a flexible ruler laid on the trunk surface, a decreased lumbar lordosis [7], and increased thoracic kyphosis [7] is reported with obesity. However, when adjusted for gender and age there was no effect of obesity on the lumbar lordosis $[7,8]$ or thoracic kyphosis [7]. Using photographs the lumbar lordosis and thoracic kyphosis are increased with obesity [9]. Using markers placed on boney landmarks, an increased anterior pelvic tilt is reported with obesity; however, there was no significant difference in the lumbar lordosis and thoracic kyphosis angles $[10,11]$. Using radiographs showed increased lumbar lordosis; thoracic kyphosis and sacrum slant angle are seen with obesity and further increased when the adipose tissue was more centrally distributed around the trunk [12]. The lumbar lordosis is reported to be positively correlated with body mass index (BMI) although there was no significant correlation for the thoracic kyphosis [13]. However, Nourbakhsh et al. [14] report a negative correlation between lumbar lordosis and weight.

The differences in the literature may be related to methodological approaches. Obesity is characterized by additional tissue over the underlying skeleton that is not distributed uniformly. Thus surface measurement approaches following the outline of the skin may not represent the underlying spine. Palpation errors in determining boney landmarks can also significantly affect the calculated postural angles 
[15]. Radiographic approaches, while being able to image the spine [12] and thus a more direct measure is allowed, have significant issues in relation to participation safety. Some studies include participants with low back pain [12]; however, it is unclear whether the obesity is the cause of the postural change or a cofactor. It is also possible that obesity does not have a consistent effect on standing posture as the spinal curves are the results of not only the boney vertebral structure but also the trunk musculature and active postural strategies may be used. During pregnancy, where there is also increased abdominal mass, a flattening of the lumbar lordosis, rather than an increase, is reported for some women $[16,17]$. The strategy of flattening of the lumbar lordosis is thought to reduce the moment arm length of the abdominal weight force and thus reduce the mechanical effect of the anterior moment [16]. The postural strategy is not seen in all women nor is it consistent over the pregnancy and therefore the response is thought to be individual $[16,17]$. Such a response may also be seen in obese participants and may be an explanation for the differences in the literature. Statistical approaches that are based on individual responses rather than being based on group means (which mask mathematical variations in opposite directions) may be useful in further research to elucidate the effect of individual responses.

\section{Postural Control and Balance}

Balance during static and dynamic tasks is an important factor in the successful completion of many daily activities. People with obesity are shown to have poorer static balance control [18-22], and this is further exacerbated when the eyes are closed [18-20,23] and information other than vision must be used to maintain balance. The underlying reasons for the poor balance control with increased mass have been the subject of several investigations. Poor lower limb strength has been proposed [21, 24]; however, individuals with high lower limb strength as well as high mass also showed poor static balance control [20] in agreement with Handrigan et al. [25], who demonstrated weight loss was more important in balance control than strength. It is possible that plantar mechanoreceptors may be less receptive due to the continuous pressure of supporting a large mass [21, 22] and are therefore less able to contribute to the control of balance.

Balance control may also be affected by the distribution of adiposity such as that seen in the typical patterns of adiposity differences between genders [24] although the literature is equivocal [19]. Balance is poor in an anteroposterior direction but not in a mediolateral direction in women $[23,24]$. In men balance is poor in both anteroposterior and mediolateral directions where typically the mass distribution is such that the center of mass is located higher in the body and therefore generates a larger torque around the base of support [24]. Cruz-Gómez et al. [19], however, report no difference between genders. The differences in results may be due to methodological differences, as when comparisons are made only between genders then no difference is seen [24]. It is also possible that differences in the literature may be related to the participants. While genders have typical patterns of adiposity there are variations and, therefore, the inclusion of a waist-hip ratio as well as weight to account for mass distribution is recommended for further research into the effects of obesity [24].

Much of the literature has focused on static balance control; however, falls typically result from a postural perturbation [26]. The increased inertia as a result of the increased mass may be protective from perturbations where the initial inertia must be overcome [26, 27]. However, when an initial angular velocity was imposed balance recovery using an ankle strategy was poor, suggesting that the type of perturbation was important in balance recovery with obesity [27].

Compensatory strategies to achieve a task may also be an issue in balance control with increased weight. Increased girth dimensions in relation to a normal weight base support dimensions afforded by the foot makes postural control already more difficult [23]. However, obese individuals place themselves more at risk of losing balance by moving the center of mass closer to the edge of the base of support when asked to undertake an aiming task [28].

\section{Range of Motion of the Trunk Segments}

Obese individuals have reported functional limitations in activities of daily living, particularly for tasks requiring trunk and hip flexibility [29]. The required range of motion (ROM) of the trunk and its segments to accomplish a daily task varies with the task; therefore, an important question is to first understand the extent of the mechanical limitations in the affected joints or segments ROM. In sitting, forward flexion ROM of the thoracic segment [30•] and thoracolumbar spine [30॰] is decreased for obese participants with no change in change in pelvic segment [30•] and hip joint motion [30•]. In standing, forward flexion ROM of the trunk segment $[10,11]$, thoracic segment $[10,11,30 \bullet]$, and thoracolumbar spine is decreased $\left[10,30^{\bullet}\right]$ with obesity, with no change in change in pelvic segment $[10,30 \cdot$ and hip joint [30•] forward flexion ROM. Lateral flexion ROM of the trunk segment in standing is not significant altered with obesity $[10,11]$; however, the thoracic segment ROM decreased $[10,11]$ while the pelvic segment ROM showed no significant difference $[10,11]$. Park et al. [31] reported lumbar spine extension is decreased; however, contrary to other reports lumbar spine lateral flexion is decreased.

Trunk forward flexion motion is restricted in both sitting and standing, in line with reduced hip joint flexion 
magnitude when supine [4]. Obstruction to the seated movement would be expected as the excessive anterior trunk tissue is located adjacent to the thighs when seated on a chair. Apposition between the anterior thigh and the abdominal tissue would restrict the forward flexion motion. Standing forward flexion and lateral bending, however, although not mechanically obstructed by the thighs, may be difficult to perform owing to decreased forward stability. Difficulties with balance [29] may make obese people more cautious in moving to the end of range.

The reduced forward flexion and lateral bending motion of the trunk is not seen across all segments and joints. Pelvic segment displacement $\left[10,30^{\bullet}\right]$ and hip joint ROM [30•] for both seated and standing motion are similar in normal weight and obese groups. Therefore, excessive trunk tissue does not mechanically alter pelvic segment forward flexion motion. Trunk forward flexion, however, is achieved by a combination of spinal and pelvic segment motion, with part of the motion accomplished by reducing the distance between the thoracic cage and the pelvis. It is possible, similar to pregnancy [32], that the excessive anterior trunk tissue provided a physical obstruction to the flexion motion of the thoracic segment with a consequent reduction in thoracic segment $\left[10,30^{\bullet}\right]$ and thoracolumbar spinal motion [30 $]$. Also the higher the BMI, the more the angular displacement of the thoracic segment and thoracolumbar spine ROM is decreased [30॰], indicating that increasing adiposity will lead to further motion restriction. The differing response to changed motion in the different segments of the trunk highlight the requirements for future investigations to consider the potential differing responses of the trunk segments and the pelvis when investigating the effects of obesity on trunk motion rather than treat the trunk as a single segment.

Thoracic segment-reduced ROM with normal pelvic segment ROM is generally seen under conditions of increased mass and trunk girths such as in obesity and late pregnancy across forward flexion, lateral flexion, and trunk axial rotation $[10,30 \bullet, 32]$. While the etiology of these differential changes is not understood, differing effects on individual trunk segments may also lead to altered movement patterns within the trunk, which may affect the musculoskeletal demands on the segment and thus may be a factor in back pain etiology. The main factor in vertebral osteophyte development is obesity [33], with the implication that the relationship is due to increased mass. However, evidence for differential effects on trunk segment loads is seen in the stronger relationship, between increased body mass and the frequency of vertebral osteophytes, in the thoracic region in comparison to that in the lumbar spine [34]. Saberi et al. [35] also show no relationship between nucleus pulposus dislodgement and modic changes in the lumbar spine with obesity. Further research is required to investigate the mechanical effects of differential effects of obesity on trunk segment ROM and back pain etiology and spinal morphological changes.

\section{Upper Limb Motion}

While there has been much literature focus on the effects of obesity on the trunk and overall whole body motion, motion of the upper limb is also affected by obesity. Shoulder extension and adduction are significantly reduced due to obstruction by adipose tissue [31]. For goal-directed aiming movements, such as reaching for a switch, there is increased movement time, a longer deceleration phase, and decreased aiming velocity when standing [36], indicating that the additional inertial load related to the increased mass of the upper limb is more difficult to control [28]. This effect is not seen when seated and it is possible that sitting may provide more stable posture from which to perform the task [36].

Obesity is also associated with the onset of carpel tunnel syndrome (CTS) [37-39] although increased obesity is not predictive of CTS onset [39]. It is possible that poor positioning of the upper limb due to mechanical constraints at upper limb joints or fatigue related to holding the additional upper limb mass result in poor wrist posture with a consequential additional load on the wrist tendons. It is also possible that obesity affects the anatomy of the hand with additional adipose tissue altering the dimensions of the hand and thus affecting the ability to grasp. Further research is required to more fully understand the relationship between the onset of CTS and obesity.

\section{Working Posture}

While there is a growing body of literature surveying the effects of obesity and work-based tasks, there is a paucity of literature investigating the mechanical effects of obesity on working posture. Gilleard and Smith [30•] reported for a simulated standing work task that the obese showed a significantly more flexed posture for the thoracic and pelvic segments, the thoracolumbar spine, and the hip joint, and the hip joint net moment was also significantly larger. During the initial quiet standing posture, the obese group stood further back from the bench with the hip-to-bench distance significantly larger compared to normal weight subjects [30•], potentially because the excess abdominal tissue precluded them from standing closer to the bench. As a consequence, to complete the task, the obese group showed a more flexed posture of both the thoracic and pelvic segments, which was reflected in the hip and thoracolumbar spine postural adaptations. There was a high correlation indicating that as BMI increased, the posture was more flexed, hip joint moment increased, and the hipto-bench distance was increased [30•]. The increased hip joint moment was seen even though the moment was 
normalized to body weight and height [30•]; therefore, the increase was likely to have been the result of postural changes rather than increased body mass.

While there is a focus in the literature on the effects of increased mass, postural adaptations due to changes in body dimensions are an important effect of obesity and may have implications for further understanding the etiology of musculoskeletal pain in obesity and the effects may be task- and body segment-specific. While Peltonen et al. [40] found self-reported work-restricting pain in the neck area, back area, and hip joints is more common in obese people, Yildirim et al. [41] found no correlation between obesity and neck pain in computer users. Further research is required to investigate the mechanical effect of obesity with work-based tasks.

\section{Obesity and Rising from a Chair}

Rising from a chair is more difficult with obesity [5] and rise time is increased [42]. When rising from a chair, obese participants use a more posterior foot placement [43, 44], and a reduced trunk flexion [43-45]. Knee extension moments are increased [43-45] and the hip extension moment is decreased [43-45].

Rising to stand from a chair is a dynamic motion requiring the body's center of mass to be moved over the feet before it then rises. A more posterior foot placement ensures that the body center of mass is closer to the foot and therefore less effort is required to move the mass anteriorly and achieve a successful rise to stand with obesity.

The reasons for the reduced trunk flexion are unclear. Decreased trunk flexion may be related to physical apposition with the thighs due to abdominal adiposity. However, the total percent body fat was shown to be a better predictor of a decrease in chair rise functionality rather than the distribution of fat between abdomen and lower limbs [6], which indicates physical apposition is not the primary cause. It is also possible that, as seen in pregnancy, the increased mass of the trunk with obesity may be an advantage when rising to stand [46]. Forward trunk flexion generates horizontal linear momentum, which is essential for rising to stand [47]. As the trunk mass is increased, sufficient horizontal linear momentum may have been generated for a successful rise.

Control of horizontal linear momentum is required to maintain upright stance at end of the motion; therefore, a strategy to minimize the horizontal momentum would be required if balance is to maintained at the end of the motion. The three studies [43-45] investigating the effect of obesity on the mechanics of rising to stand from a chair used a controlled task with arms folded across the chest and a rise to stance. Therefore, a minimized horizontal momentum would be used as a strategy particularly with obesity where difficulties with balance have been reported [29]. Further studies are required to investigate the mechanics of the task when participants are required to rise to stand from a chair using their upper limbs in a normal manner and walk forward.

The literature suggests that the decreased hip extension moment is a strategy to reduce the load on the lower back with a consequential increase in knee extension moment and increased load on the knee [43-45]. However, these studies included obese participants with lower back pain [43, 45] and low back pain status was not reported [44] in comparison to control groups with no lower back pain. For participants with no lower back pain, Gilleard et al. [46] showed no significant trends for change in hip and knee extension moments as pregnancy progressed. Figures shown in Sibella et al. [44] also indicate that the peak knee and hip moment occurs earlier in the obese group in comparison to controls although no time of peak moment nor knee joint extension velocity data was presented. The thigh is the major contributor to the vertical linear momentum of the center of mass during rising to stand [48]; therefore, further research is required to understand the movement strategies used by obese participants in the absence of low back pain.

\section{Obesity and Lifting}

There is a paucity of literature on the effects of obesity on the mechanics of lifting. While holding a box in a static posture when obese results in higher perceived exertion [49], obesity does not affect the maximum acceptable weights of lift [50]. Xu et al. [51•] reported that obesity significantly increased the lumbar spine transverse plane rotation and sagittal plane velocity and acceleration when lifting. There was no effect on peak transverse plane rotation, peak sagittal plane displacement, or the ground reaction forces (GRFs) when normalized to mass. There was no effect of obesity on the variability of the lifting kinematics [51•] .

It is not clear why an increase in body mass results in a lifting technique that increases the inertial load on the spine. There was no evidence that the obese participants had greater strength [51•], however, it is possible that the two groups used a different lifting strategy. The obese participants may have used an approach that utilized an inertial technique to raise the box (stoop lift approach) rather than a semi-squat lift. As knee flexion ROM is significantly reduced with obesity [31], a squat or semi-squat lift requiring significant knee flexion may be more difficult than a stoop lift approach. The additional inertial mass of the trunk and upper segment may be an advantage when using the stoop lift technique. While for functional task such as rising to stand from a chair, and stair ascent and descent as obesity increases the task are more affected, class of obesity does not affect lifting mechanics any further [52•]. Further 
research is required to investigate the mechanical effect of obesity on the selected lifting techniques.

\section{Obesity and Walking}

Walking is an important functional task that underpins work, leisure, and daily tasks. However, the literature is equivocal on the effects of obesity on the kinematics and kinetics of walking. Slower velocity [52•, 53-55], reduced cadence $[52 \cdot 54,55]$, reduced stride length [53-56], reduced swing time [55, 57], increased stance time $[53,55,57]$, decreased single support time [53, 56], and increased double support time [53, 58] have been reported. In contrast, no change in velocity [56-60], cadence [57, 58, 61], step length [52•, 58, 59], stride length [61], stance time [57], single leg support time [52•], double leg support time [52•], and swing phase duration [52•] have also been reported. The literature is consistent for increased step width [54, 56, 58, 59, 61]. Błaszczyk et al. [57] have reported increased leg swing velocity, which was positively correlated to BMI.

Increased peak hip joint flexion [62], extension [55], and sagittal plane ROM [63], and increased ankle eversion from mid stance to pre-swing [53] have been reported. In contrast, no change in hip joint sagittal plane ROM [62], ankle joint peak, or ROM of eversion [62] has also been reported. The literature is consistent showing increased hip adduction during terminal stance and pre-swing $[53,62]$, and increased knee adduction in stance and swing [53, 62]. Increased ankle plantar flexion [55] and reduced knee flexion [55] have also been reported.

Lai et al. [53] reported decreased second vertical peak GRF and propulsive GRF not related to mass. However, Browning et al. [58] reported no difference in vertical, anterior posterior, or mediolateral GRF when mass was accounted for. The transverse coefficient of friction was reported to be decreased with no change in the anteriorposterior coefficient of friction [59].

When mass is accounted for there is no difference in hip flexion moment [55]; however, a lower knee extensor moment [55] and a lower peak ankle plantarflexor moment [53, 55] is reported. An increase of ankle inversion moment not related to mass [53] has also been reported. In line with these findings there is also no difference in external mechanical work when adjusted for mass [58, 64].

The literature is equivocal on the effect of increased mass as against the effect of increased girths on gait. The increased step width is thought to be an obstructive mechanical issue related to the increased thigh girth $[53,65]$ and it is possible that increased hip and knee adduction are a consequential effect of the increased step width. However, the increased knee adduction $[53,62]$ is reported to be related to weight, not increased thigh girth [66].

Strategies such as slower velocity, shorter step length, wider base of support, and longer time in stance are similar to adaptations seen in late pregnancy [67]. These may be a successful strategy for increasing stability [53, 59, 67], supporting the finding that when investigated prospectively obesity does not increase overall fall risk [68]. However, use of these stability strategies when walking, coupled with the finding that people who are obese undertake less ambulatory activity during their daily lives [69], is important information for developing strategies used to reduce sedentary lifestyle and increase physical activity with a view toward the regulation of weight [70].

Methodological approaches, and the walking task undertaken may underpin the differences in the literature. While the necessary technology restricts much of the gait data collection to traditional laboratories $[53-56,58,59,62$, 63], wearable devices have more recently allowed movement data collection over a 24 -hour period [52•]. The scope of data collection variables is limited; however, the data reflects a more realistic scenario than the constraints imposed by use of treadmills or walkways. Familiarization with the environment is important and while familiarization methods are sometimes reported $[55,58]$ many do not report how the participants practiced the given task [53, 54, 62]. The walking task also varies, with some studies using preferred walking speed $[53-55,59,63,64]$ and others a predetermined speed $[55,58,62]$. Further research is required using wearable devices to more fully understand the effects of obesity on gait in daily activities where participants are using their normal gait habits.

Another confounding factor when comparing studies is the use of participants with varying levels of obesity. Some studies included participants with $\mathrm{BMI}>30 \mathrm{~kg} / \mathrm{m}^{2}[53,59$, $62,63]$, another used BMI $>35 \mathrm{~kg} / \mathrm{m}^{2}$ [54], BMI average $39.6 \mathrm{~kg} / \mathrm{m}^{2}$ [64], and BMI ranging from 30 to $51.4 \mathrm{~kg} / \mathrm{m}^{2}$ [52.]. The delineation of future studies to particularly classes of obesity is important to more fully understand the effects of obesity, as effects may be independent of the magnitude of obesity while others may be related.

\section{Conclusions}

Obesity is characterized by increased mass and dimensions. Functional task limitations and changes in the mechanical strategies used to complete functional tasks may be related independently to increased mass or increased dimensions and the response may also be individualized. Increased mass is not always detrimental. In lifting the increased mass appears to be used positively as part of the strategy of the lifting technique utilized. The mechanical strategies may 
vary with the class of obesity or mass distribution to the trunk or lower limbs. Much of the literature is equivocal and it is possible that methodological approaches and class of obese participants result in different or conflicting outcomes. Future investigations into mechanical underpinnings of the functional task limitations need to consider the class of obesity within the study group.

Disclosure No potential conflicts of interest relevant to this article were reported.

\section{References}

Papers of particular interest, published recently, have been highlighted as:

- Of importance

1. Backholer K, Wong E, Freak-Poli R, et al. Increasing body weight and risk of limitations in activities of daily living: a systematic review and meta-analysis. Obes Rev. 2012;13(5):456-468.

2. Houston DK, Ding J, Nicklas BJ, et al. The association between weight history and physical performance in the Health, Aging and Body Composition study. Int J Obes. 2007;31:16807.

3. Vincent HK, Ben-David K, Conrad BP, et al. Rapid changes in gait, musculoskeletal pain, and quality of life after bariatric surgery. Surg Obes Relat Dis. 2012;8(3):346-354.

4. Escalante A, Lichtenstein MJ, Dhanda R, et al. Determinants of hip and knee flexion range: results from the San Antonio Longitudinal Study of Aging. Arthritis Care Res. 1999;12:818.

5. Vincent HK, Vincent KR, Lamb KM. Obesity and mobility disability in the older adult. Obes Rev. 2010;11:568-79.

6. Foster NA, Segal NA, Clearfield JS, et al. Central versus lower body obesity distribution and the association with lower limb physical function and disability. PM R. 2010;2:1119-26.

7. Lang-Tapia M, España-Romero V, Anelo J, et al. Differences on spinal curvature in standing position by gender, age and weight status using a noninvasive method. J Appl Biomech. 2011;27:14350 .

8. Youdas JW, Hollman JH, Krause DA. The effects of gender, age, and body mass index on standing lumbar curvature in persons without current low back pain. Physiother Theory Pract. 2006;22:229-37.

9. Fabris De Souza SA, Faintuch J, Valezi AC, et al. Postural changes in morbidly obese patients. Obes Surg. 2005;15:1013-6.

10. Vismara L, Menegoni F, Zaina F, et al. Effect of obesity and low back pain on spinal mobility: a cross sectional study in women. J NeuroEng Rehabil. 2010;7:3-11.

11. Menegoni F, Vismara L, Capodaglio P, et al. Kinematics of trunk movements: protocol design and application in obese females. $\mathrm{J}$ Appl Biomater Biomech. 2008;6:178-85.

12. Guo JM, Zhang GQ, Alimujiang. Effect of BMI and WHR on lumbar lordosis and sacrum slant angle in middle and elderly women. China J Orthop Traumatol. 2008;21:30-1.

13. Hoseinifar M, Ghiasi F, Akbari A. The relationship between lumbar and thoracic curves with body mass index and low back pain in students of Zahedan University of Medical Sciences. J Med Sci. 2007;7:984-90.
14. Nourbakhsh MR, Moussavi SJ, Salavati M. Effects of lifestyle and work-related physical activity on the degree of lumbar lordosis and chronic low back pain in a middle east population. J Spinal Disord. 2001;14:283-92.

15. Moriguchi CS, Carnaz L, Silva LCCB, et al. Reliability of intraand inter-rater palpation discrepancy and estimation of its effects on joint angle measurements. Man Ther. 2009;14:299-305.

16. Moore K, Dumas GA, Reid JG. Postural changes associated with pregnancy and their relationship with low-back pain. Clin Biomech. 1990;5:169-74.

17. Gilleard WL, Crosbie J, Smith R. Static trunk posture in sitting and standing during pregnancy and early postpartum. Arch Phys Med Rehabil. 2002;83:1739-44.

18. Dutil M, Handrigan GA, Corbeil P, et al. The impact of obesity on balance control in community-dwelling older women. Age 2012:1-8. doi:10.1007/s11357-012-9386-x

19. Cruz-Gómez NS, Plascencia G, Villanueva-Padrón LA, et al. Influence of obesity and gender on the postural stability during upright stance. Obes Facts. 2011;4:212-7.

20. Handrigan GA, Berrigan F, Hue O, et al. The effects of muscle strength on center of pressure-based measures of postural sway in obese and heavy athletic individuals. Gait Posture. 2012;35:88-91.

21. Hue O, Simoneau M, Marcotte J, et al. Body weight is a strong predictor of postural stability. Gait Posture. 2007;26:32-8.

22. Teasdale N, Hue O, Marcotte J, et al. Reducing weight increases postural stability in obese and morbid obese men. Int $\mathrm{J}$ Obes. 2007;31:153-60.

23. Mainenti MRM, de Carvalho Rodrigues É, de Oliveira JF, et al. Adiposity and postural balance control: correlations between bioelectrical impedance and stabilometric signals in elderly Brazilian women. Clinics. 2011;66:1513-8.

24. Menegoni F, Galli M, Tacchini E, et al. Gender-specific effect of obesity on balance. Obesity. 2009;17:1951-6.

25. Handrigan G, Hue O, Simoneau M, et al. Weight loss and muscular strength affect static balance control. Int J Obes. 2010;34:936-42.

26. Miller EM, Matrangola SL, Madigana ML. Effects of obesity on balance recovery from small postural perturbations. Ergonomics. 2011;54:547-54.

27. Matrangola SL, Madigan ML. The effects of obesity on balance recovery using an ankle strategy. Hum Mov Sci. 2011;30:584-95.

28. Berrigan F, Simoneau M, Tremblay A, et al. Influence of obesity on accurate and rapid arm movement performed from a standing posture. Int J Obes. 2006;30:1750-7.

29. Larsson UE, Mattsson E. Functional limitations linked to high body mass index, age and current pain in obese women. Int $\mathrm{J}$ Obes. 2001;25:893-9.

30. - Gilleard W, Smith T. Effect of obesity on posture and hip joint moments during a standing task, and trunk forward flexion motion. Int J Obes. 2007;31:267-71. Investigates the mechanical effects of obesity on a work task.

31. Park W, Ramachandran J, Weisman P, et al. Obesity effect on male active joint range of motion. Ergonomics. 2010;53:102-8.

32. Gilleard W, Crosbie J, Smith R. Effect of pregnancy on trunk range of motion when sitting and standing. Acta Obstet Gynecol Scand. 2002;81:1011-20.

33. Klaassen Z, Tubbs RS, Apaydin N, et al. Vertebral spinal osteophytes. Anat Sci Int. 2011;86:1-9.

34. O'Neill TW, McCloskey EV, Kanis JA, et al. The distribution, determinants, and clinical correlates of vertebral osteophytosis: a population based survey. J Rheumatol. 1999;26:842-8.

35. Saberi H, Rahimi L, Jahani L. A comparative MRI study of upper and lower lumbar motion segments in patients with low back pain. J Spinal Disord Tech. 2009;22:507-10.

36. Berrigan F, Hue O, Teasdale N, et al. Obesity adds constraint on balance control and movement performance. In: 52nd Human 
Factors and Ergonomics Society Annual Meeting: 2008; New York, NY; 2008: 1364-8.

37. Forst L, Friedman L, Shapiro D. Carpal tunnel syndrome in spine surgeons: a pilot study. Arch Environ Occup Heal. 2006;61:259-62.

38. Burt S, Crombie K, Jin Y, et al. Workplace and individual risk factors for carpal tunnel syndrome. Occup Environ Med. 2011;68:928-33.

39. Gell N, Werner RA, Franzblau A, et al. A longitudinal study of industrial and clerical workers: incidence of carpal tunnel syndrome and assessment of risk factors. J Occup Rehabil. 2005; 15:47-55

40. Peltonen M, Lindroos AK, Torgerson JS. Musculoskeletal pain in the obese: a comparison with a general population and long-term changes after conventional and surgical obesity treatment. Pain. 2003;104:549-57.

41. Yildirim Y, Gelecek N, Özcon A, et al. The risk factors contributing to neck pain in computer users. Bilgisayar kullananlarda boyun a risina etki eden risk faktörleri. 2004;15:114-9.

42. Davis JW, Ross PD, Preston SD, et al. Strength, physical activity, and body mass index: relationship to performance-based measures and activities of daily living among older Japanese women in Hawaii. J Am Geriatr Soc. 1998;46:274-9.

43. Bertocco P, Baccalaro G, Montesano A, et al. The analysis of sitto-stand movement in obese and normal subjects: biomechanic evaluations and postural changes between groups. Europa Medicophysica. 2002;38:131-7.

44. Sibella F, Galli M, Romei M, et al. Biomechanical analysis of sitto-stand movement in normal and obese subjects. Clin Biomech. 2003;18:745-50.

45. Galli M, Crivellini M, Sibella F, et al. Sit-to-stand movement analysis in obese subjects. Int J Obes. 2000;24:1488-92.

46. Gilleard W, Crosbie J, Smith R. A longitudinal study of the effect of pregnancy on rising to stand from a chair. J Biomech. 2008;41:779-87.

47. Pai YC, Naughton B, Chang R, et al. Control of body centre of mass momentum during sit-to-stand among young and elderly adults. Gait Posture. 1994;2:109-16.

48. Pai YC, Rogers MW. Segmental contributions to total body momentum in sit-to-stand. Med Sci Sports Exerc. 1991;23:225-30.

49. Park W, Singh DP, Levy MS, et al. Obesity effect on perceived postural stress during static posture maintenance tasks. Ergonomics. 2009;52:1169-82.

50. Singh D, Park W, Levy MS. Obesity does not reduce maximum acceptable weights of lift. Appl Ergon. 2009;40:1-7.

51. - Xu X, Mirka GA, Hsiang SM. The effects of obesity on lifting performance. Appl Ergon. 2008;39:93-8. Reports a positive effect of obesity on a functional task.

52. - Benedetti MG, Di Gioia A, Conti L, et al. Physical activity monitoring in obese people in the real life environment. J NeuroEng Rehabil. 2009;6:47-56. Reports mechanical data from study of daily lives rather than laboratory-based.
53. Lai PPK, Leung AKL, Li ANM, et al. Three-dimensional gait analysis of obese adults. Clin Biomech. 2008;23:S2-6.

54. De Souza SAF, Faintuch J, Valezi AC, et al. Gait cinematic analysis in morbidly obese patients. Obes Surg. 2005;15:1238-42.

55. DeVita P, Hortobágyi T. Obesity is not associated with increased knee joint torque and power during level walking. J Biomech. 2003;36:1355-62.

56. Vismara L, Bertocco P, Parisio C, et al. Gait analysis on a treadmill in young, obese women. J Appl Biomater Biomech. 2006;4:215-6.

57. Błaszczyk JW, Plewa M, Cieślińska-Świder J, et al. Impact of excess body weight on walking at the preferred speed. Acta Neurobiol Exp. 2011;71:528-40.

58. Browning RC, McGowan CP, Kram R. Obesity does not increase external mechanical work per kilogram body mass during walking. J Biomech. 2009;42:2273-8.

59. Wu X, Lockhart TE, Yeoh HT. Effects of obesity on slip-induced fall risks among young male adults. J Biomech. 2012;45(6):1042-1047.

60. Browning RC, Kram R. Energetic cost and preferred speed of walking in obese vs. normal weight women. Obes Res. 2005;13:891-9.

61. Browning RC, Kram R. Effects of obesity on the biomechanics of walking at different speeds. Med Sci Sports Exerc. 2007;39:163241.

62. Russell EM, Hamill J. Lateral wedges decrease biomechanical risk factors for knee osteoarthritis in obese women. J Biomech. 2011;44:2286-91.

63. Smith B, Roan M, Lee M. The effect of evenly distributed load carrying on lower body gait dynamics for normal weight and overweight subjects. Gait Posture. 2010;32:176-80.

64. Malatesta D, Vismara L, Menegoni F, et al. Mechanical external work and recovery at preferred walking speed in obese subjects. Med Sci Sports Exerc. 2009;41:426-34.

65. Spyropoulos P, Pisciotta JC, Pavlou KN. Biomechanical differences in obese, nonobese men's gait. J Am Osteopath Assoc. 1992;92:36.

66. Segal NA, Yack HJ, Khole P. Weight, rather than obesity distribution, explains peak external knee adduction moment during level gait. Am J Phys Med Rehabil. 2009;88:180-91.

67. Lymbery JK, Gilleard W. The stance phase of walking during late pregnancy: temporospatial and ground reaction force variables. J Am Podiatr Med Assoc. 2005;95:247-53.

68. Rosenblatt NJ, Grabiner MD. Relationship between obesity and falls by middle-aged and older women. Arch Phys Med Rehabil 2012;93:718-22.

69. Levine JA, Lanningham-Foster LM, McCrady SK, et al. Interindividual variation in posture allocation: possible role in human obesity. Science. 2005;307:584-6.

70. Marshall SJ, Ramirez E. Reducing sedentary behavior: a new paradigm in physical activity promotion. Am J Lifestyle Med. 2011;5:518-30. 